OPINION

\title{
Horizontal gene transfer in osmotrophs: playing with public goods
}

\section{Thomas A. Richards and Nicholas J. Talbot}

Abstract | Osmotrophic microorganisms, such as fungi and oomycetes, feed by secreting depolymerizing enzymes to process complex food sources in the extracellular environment, and taking up the resulting simple sugars, micronutrients and amino acids. As a consequence of this lifestyle, osmotrophs engage in the acquisition and protection of public goods. In this Opinion article, we propose that horizontal gene transfer (HGT) has played a key part in shaping both the repertoire of proteins required for osmotrophy and the nature of public goods interactions in which eukaryotic microorganisms engage.

Osmotrophic microorganisms feed by secreting extracellular depolymerizing enzymes into the environment to degrade complex polymers, such as cellulose, lignin and proteins, and by transporting the resulting simple, monomeric sugars and amino acids into their own cells. Fungi, oomycetes and many bacteria feed by osmotrophy, and in using this trophic mechanism, these organisms have become the principal degraders of biomass in most terrestrial ecosystems, as well as important pathogens of plants and animals. One of the most important consequences of osmotrophy is the participation of microorganisms in both competitive and cooperative public goods interactions, because the food sources of these organisms reside outside the cells even as these foods are being processed, and are therefore available to others. In this Opinion article, we focus primarily on microbial eukaryotes and propose that genes encoding proteins associated with osmotrophy have been important in the evolutionary ecology and public goods interactions of these microorganisms. We also present evidence that horizontal gene transfer (HGT) has had a major role in reconfiguring osmotrophic functions in fungi and oomycetes. These results are part of a growing body of evidence which suggests that diverse evolutionary mechanisms operating on genomes have shaped social interactions across a range of microorganisms ${ }^{1-5}$.

Osmotrophic eukaryotic microorganisms The largest group of osmotrophic eukaryotic microorganisms, in terms of biodiversity, is the kingdom Fungi. According to the fossil record, fungi colonized the terrestrial environment more than 400 million years ago, in close association with early land plants ${ }^{6-9}$. Extant fungi occupy diverse ecosystems and undertake a wide variety of interactions, ranging from highly mutualistic symbioses to devastating diseases of both animals and plants ${ }^{10}$. Fungi are diverse in terms of species complexity ${ }^{11-17}$, gene repertoire and biochemical capabilities ${ }^{18-24}$, and form a range of cell types, including unicellular yeasts, motile flagellated zoospores and polarized multicellular hyphae. They also form specialized feeding structures, such as appressoria (FIG. 1a), haustoria and rhizoid structures (FIG. 1 b), which can go on to form complex tissues within multicellular fruiting bodies ${ }^{12}$. It is clear that early in the diversification of fungi, the ability to carry out phagocytosis - a mode of feeding that generates private goods was lost, and the overwhelming majority of extant fungi are instead dependent on osmotrophy ${ }^{13}$, a process that generates both public and club goods.

Osmotrophy has proved to be a successful feeding strategy for fungi in particular, and has allowed them to colonize diverse heterogeneous terrestrial environments where nutrients are plentiful but largely inaccessible to most competitors because they take the form of complex biological molecules such as cellulose and lignin (for example, within leaf litter or soil) or the cellulose- and protein-rich tissues of plant and animal hosts, respectively. As a consequence, fungi have evolved to become important decomposers of biomass in most terrestrial ecosystems ${ }^{25}$. Obligate osmotrophy is, however, not unique to fungi. Many bacteria, for instance, feed in an analogous manner, and other eukaryotic groups, such as hyphochytriomycetes (FIG. 1c) and oomycetes (FIG. 1d) (sometimes collectively termed the pseudofungi ${ }^{26}$ ), also feed osmotrophically and adopt filamentous growth habits, allowing invasive growth in heterogeneous substrates. Importantly, these eukaryotes also lost the ability to carry out phagotrophy and became obligately osmotrophic ${ }^{26,27}$.

Osmotrophy has a number of distinct advantages as a feeding strategy. External digestion of large and complex polymers allows greater control over substances that are allowed to enter a cell (FIG. 2a), thus minimizing potential routes of infection and intake of harmful substances. Furthermore, osmotrophy allows greater fidelity in nutrient acquisition, such that the repertoire of digestive enzymes and uptake transporters expressed by an osmotrophic species can be altered to match a particular need, like the colonization of a new substrate $e^{28-30}$. However, osmotrophy also carries some disadvantages or risks, such as the utilization of secreted enzymes by competitors within a community and the loss of derived nutrient sources to neighbouring microorganisms (FIG. 2b,c) or by diffusion ${ }^{30-32}$. An important consequence of osmotrophy is therefore that microorganisms which use this trophic mechanism must engage in producing, protecting and acquiring public goods $s^{33,34}$. Thus, osmotrophy-associated genes predominantly encode secreted depolymerizing enzymes and cognate transporter proteins (FIG. 2), as well as genes associated with toxin production or detoxification. Toxin production can be used by osmotrophs to exclude competitors, thereby protecting public goods from being used by others (for examples, see REFS 35,36). The acquisition or reconfiguration of these traits is likely to be favourable, as both allow microorganisms to colonize new environments and/or make use of additional metabolites, but also equip microorganisms with new capabilities with which to engage in social competition (for example, stealing bacterial siderophores ${ }^{30}$ ).

\section{Osmotrophs and public goods interactions}

As discussed above, the production of osmotrophic phenotypes represents a cost to the individual, because a fraction of the protein produced or food digested might be lost to competitors ${ }^{32,34}$. As a result, selection will tend to favour individuals that stop or minimize their production of public goods but still make use of public goods manufactured by others. Cooperative behaviour 

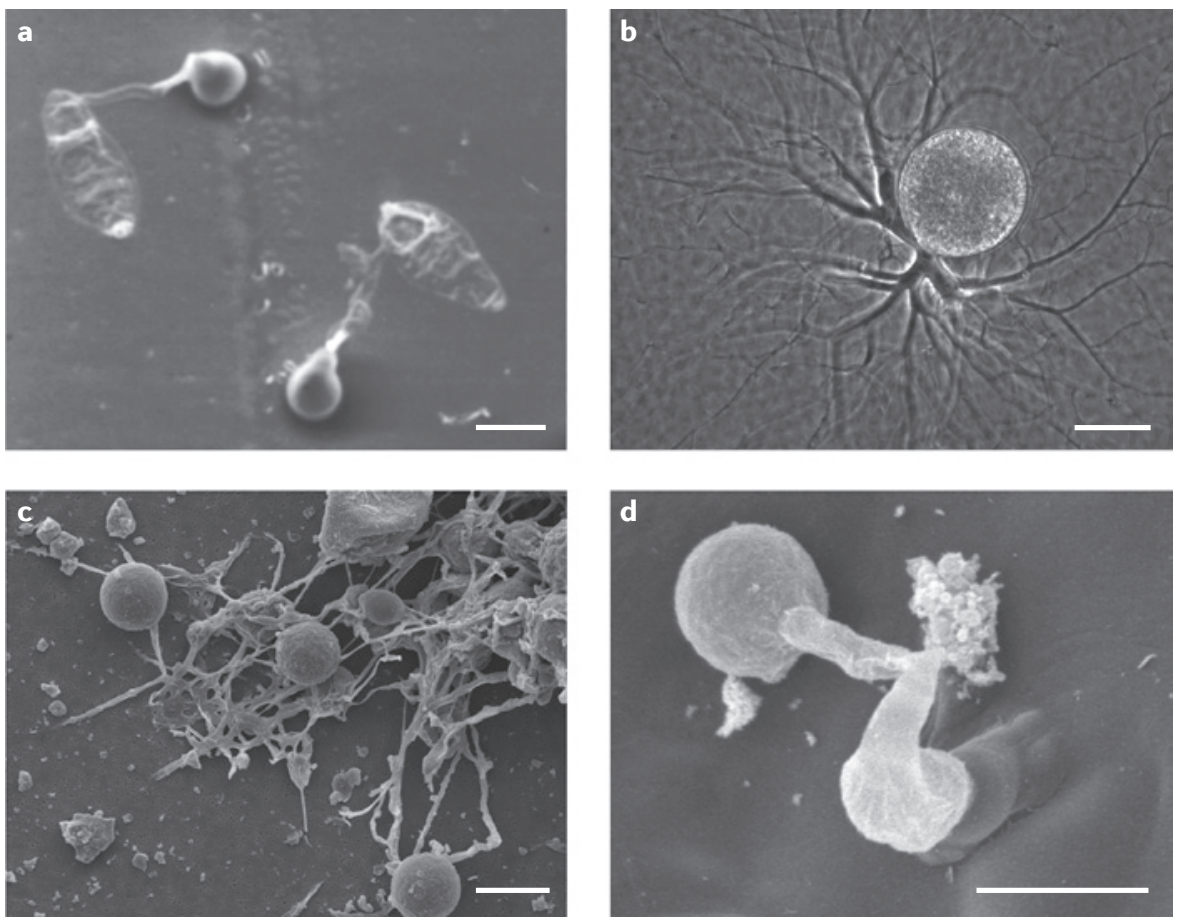

Figure 1 | The osmotrophic lifestyle in eukaryotic microorganisms. a | Scanning electron micrograph (SEM) of Magnaporthe oryzae, showing appressoria, which are specialized structures for the invasion of plant tissue. $\mathbf{b}$ | Light micrograph of sporangia and rhizoid structures of Pseudorhizidium endosporangiatum (isolate JEL 221) ${ }^{106}$.c |SEM of a fraction of a clonal multicellular assembly (or mycelium) of the pseudofungus Hyphochytrium catenoides, showing hyphal growth and interconnections. d | SEM of Phytophthora infestans attacking the leaf surface of a host plant. Scale bars represent $10 \mu \mathrm{m}$. Part b image courtesy of J. Longcore, University of Maine, USA; part d image courtesy of S. Kamoun, The Sainsbury Laboratory, UK.

can therefore give way to the emergence of cheats within a population ${ }^{34,37}$. It is generally assumed that functions are maximized if there is extensive cooperation (sometimes referred to as a 'conspiracy of doves'), but in some populations, a mixture of cooperators and cheats can be optimal ${ }^{38}$ - although even in these cases, cheats and cooperators are still in conflict. As a consequence of such conflict, it is important that osmotrophs acquire nutrients rapidly, thereby making public goods inaccessible to others, or that the organisms protect released food sources by excluding competitors from the local environment. Adaptation to these pressures can take the form of utilization of highaffinity nutrient transporters ${ }^{39-43}$, modification of the public goods produced so that they can be acquired by only a subset of the microbial community ${ }^{3,44}$, or (as mentioned above) exclusion of competitors by toxin production $^{35,36}$.

Although some metabolites generated by extracellular digestion are likely to be universally accessible (glucose or phosphate, for instance), others can be available to only a subset of the population (club goods)
- those organisms that possess a compatible uptake system (a cognate transporter protein) to take up the nutrient (FIG. 2b,c). In this way, possession of a cognate transporter might be restricted to individuals of the same species or clonal group, providing one mechanism for kin selection. However, possession of the cognate transporter by an organism of the same species that does not cooperate by helping to produce the public goods, or by a member of a different species that does not contribute to generation of the public goods, would allow such cheats to proliferate in the population (FIG. 2c). Acquiring transporters would therefore be predicted to be a means by which an individual could cheat within a population of osmotrophs.

Similarly, the secretion of enzymes that generate public goods also determines which microorganisms provide the 'work' within a population (FIG. 2b,c). For example, the secretion of invertase by Saccharomyces cerevisiae is an osmotrophic phenotype and a popular model for investigating public goods interactions $\mathrm{s}^{31,32}$. Invertase catalyses the hydrolysis of sucrose to liberate fructose and glucose outside the cell ${ }^{31}$, where a suite of hexose transporters are then utilized to recover extracellular sugars ${ }^{29,41,45}$. This osmotrophic process can result in the loss (into the environment) of up to $99 \%$ of the monosaccharides produced by invertase activity ${ }^{31}$ (but see REF. 46 for a scenario suggesting that this $99 \%$ is not all lost). The production of extracellular enzymes such as invertase clearly carries a cost to the organism, and it is therefore likely that selection favours individuals which have lost their invertase activity but still survive as cheats in a population of invertase secretors (a scenario analogous to that set out in the Black Queen hypothesis ${ }^{1}$ ). Alternatively, acquisition of activities such as invertase production might confer on a microbial lineage the ability to colonize new environments and utilize previously untapped food sources. Complex patterns of gain and loss of the genes encoding secreted depolymerizing enzymes and cognate nutrient uptake transporters are thus likely to occur within osmotrophic lineages. It is therefore clear to see how a process such as HGT might provide a means for such traits to rapidly move within a population of different species.

\section{Osmotrophic and social evolution}

There have been considerable advances in our understanding of social interactions in microbial systems (for reviews, see REFS 33,34). These interactions have been interpreted mainly using classic social evolution theory as set out by W. D. Hamilton $^{34,47,48}$, which posits that individuals gain inclusive fitness through improving the reproductive success of closely related individuals of the same species. Hamilton's rule therefore predicts that higher levels of public goods interactions will occur when individuals are within the same species group and closely related. The kin selection hypothesis for public goods interactions has received support from experimental manipulations of populations of the bacterial pathogen Pseudomonas aeruginosa, for instance, demonstrating that higher levels of siderophore production (a public goods trait for iron acquisition) evolve in communities with higher genetic relatedness ${ }^{49}$. However, it is worth noting that the spatial distribution of these interactions and the relative density of microbial communities are important factors for understanding the fate of both cheats and cooperators ${ }^{46,49-52}$. This is because the spatial distribution of a species is connected to the likelihood that related individuals will be in close proximity to each other and will therefore benefit from public goods produced by 

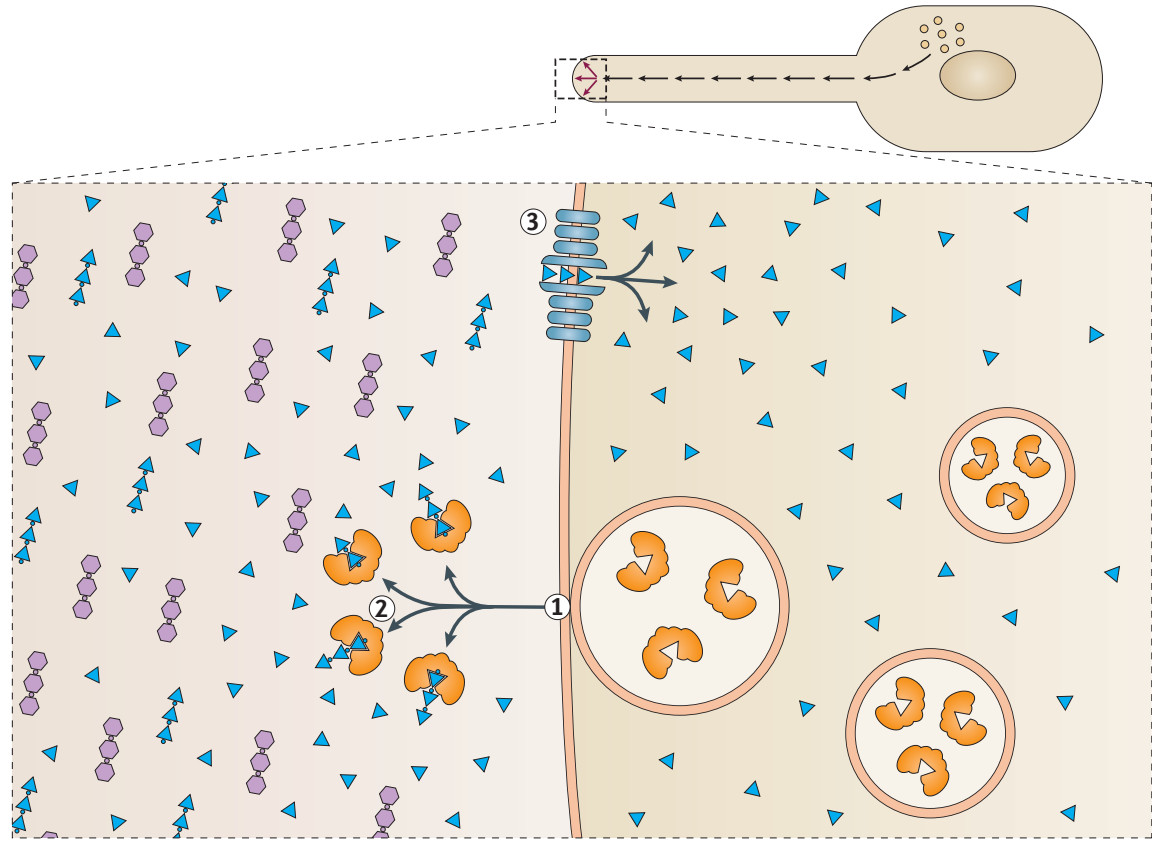

b
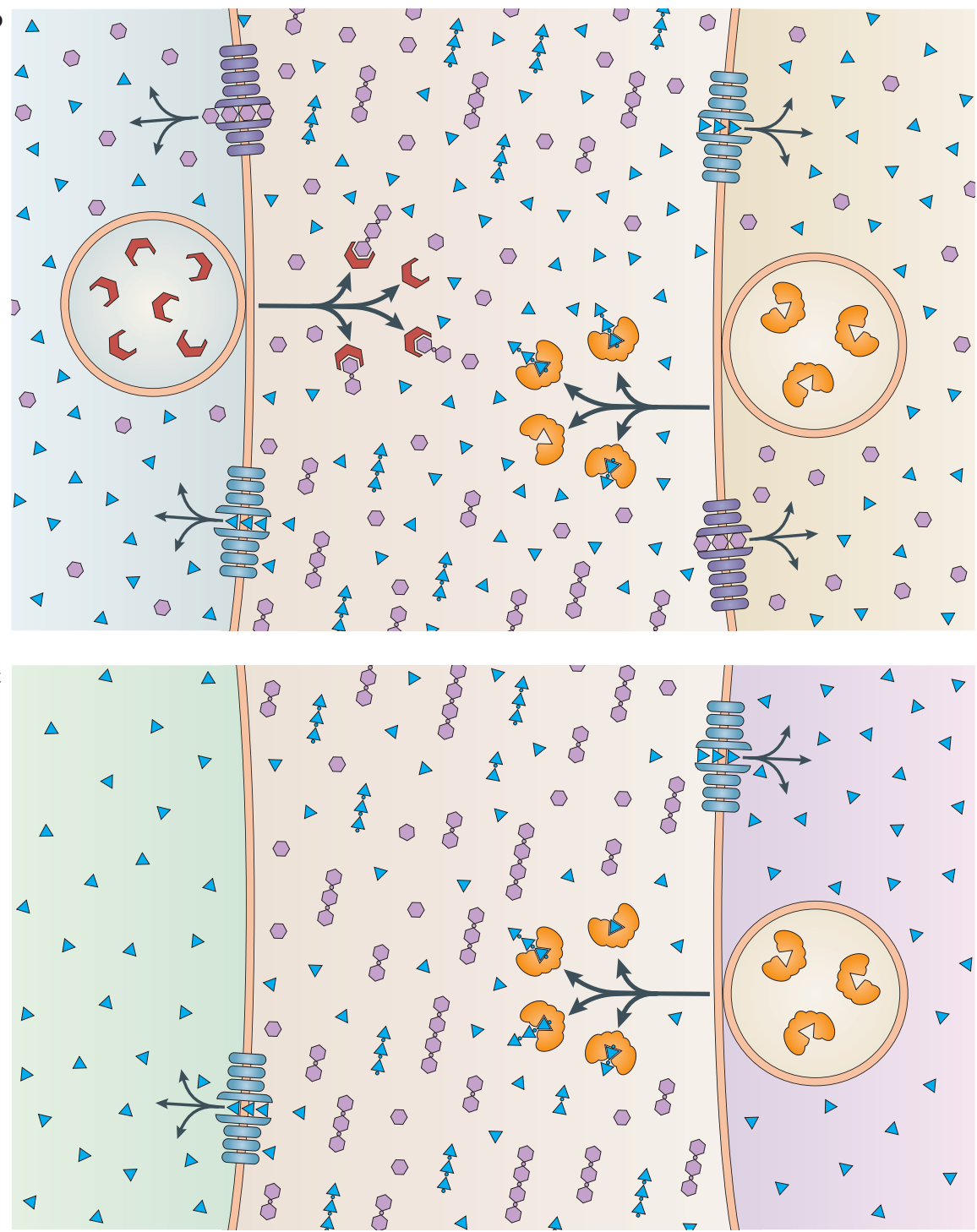

Figure 2 | Competitors and collaborators in an osmotrophic ecosystem. a|A schematic polarized cell showing adaptation to an osmotrophic function. The close-up shows a view of this polarized cell in the process of degradative enzyme secretion (step 1), breakdown or digestion of complex molecules in the extracellular environment (step 2) and selective transport of broken-down metabolites back into the cell (step 3). These are the key functions associated with osmotrophic feeding. $\mathbf{b}$ | Two cells collaborating, with each cell producing a different set of enzymes that release different club goods from which both cells can profit because both possess cognate transporter proteins. c|A situation in which one cell (right) is doing the work to make public goods available, whereas both cells are making use of the public goods because they possess the cognate transporter. The cell on the left could be described as a defector, or even a cheat.

their neighbouring kin. Microorganisms that make clonal patches (for example, fungi that develop hyphal networks or yeast colonies) produce situations in which the proximity of kin can favour public goods-producing behaviours ${ }^{46,50}$. However, there is evidence that this is not a simple rule: low levels of distribution (high viscosity) can increase local competition between relatives of the same species, meaning that intermediate dispersal (intermediate viscosity) might in many cases produce optimal situations for the evolution of public goods traits ${ }^{53-55}$. Consistent with this idea, artificial alterations in spatial structure among mycorrhizal fungi has been shown to favour cooperative fungal species ${ }^{56}$.

In these cases, kin selection operates within species, family or clonal groups, but the term 'relatedness' in these contexts often refers to whether the interacting microorganisms share compatible alleles for public goods traits, rather than average genome similarity, although generally the two are likely to be correlated unless HGT or differential gene $\operatorname{loss}^{1,2}$ has played a part. We note, however, that the species concept is difficult to apply to many microbial groups, including many fungi, that reproduce by asexual means and for which 'species groups' can contain considerable diversity in terms of sequence variation, morphology and genome content ${ }^{57-59}$. This makes the concept of kin selection equally difficult to apply to these microorganisms, and ideas like species, genetic relatedness and kin selection are therefore fluid in many microbial groups and are dependent on the distribution of certain genes rather than on patterns of vertical or familial ancestry (that is, true relatedness). 


\section{Cooperation without relatedness}

Public goods interactions are difficult to explain in systems for which genetic relatedness is low, such as communities composed of many different species, or situations in which there is no kin discrimination. Many community interactions involving osmotrophic microorganisms such as fungi and pseudofungi fall into this category, as these organisms are often found in heterogeneous communities ${ }^{16,17,25}$ such as those in soil and leaf litter.

Given the high heterogeneity of these communities, it is also important to consider alternative mechanisms that can support cooperative behaviours, such as those that enhance public goods production. These mechanisms can include reciprocal cooperative behaviour ${ }^{60,61}$, or punishment of cheats $^{62,63}$. As these features require repeated interactions or stable microbial populations, public goods production is therefore, theoretically, sustainable only in stable microbial communities composed of closely related individuals (such as family groups or individuals of the same species), which is true for certain microbial ecosystems, like hyphal networks, yeast colonies ${ }^{46,50}$ and biofilms, but less so for the communities in more heterogeneous environments. Furthermore, repeated interactions with reciprocal cooperative behaviour ${ }^{60,61}$ or punishment (sanction) of cheats ${ }^{62,63}$ are interactions that are generally more relevant for animals, although there are examples of both in non-animal systems. For example, manipulations of mycorrhizal symbiosis have demonstrated reciprocal cooperative behaviours as the plant amends the contribution of fixed carbon and the arbuscular mycorrhizal fungus amends the production of phosphate, thereby rewarding partners that make 'better' contributions to mutualism $^{64}$. Punishment of cheats is evident in plant-rhizobium mutualisms, in which bacteria that do not produce fixed nitrogen for their plant partners have been shown to have reduced reproductive success, potentially because of reduced oxygen supply from the plant host ${ }^{65}$. In this case, reduced oxygen supply represents a sanction or a punishment.

However, some theoretical work suggests that cooperation can arise when the "population density depends on the average population payoff” (REF. 66). For example, voluntary participation can be an important factor for driving public goods interactions, producing cooperative behaviour without spatial population structures and between groups of different species or subspecies ${ }^{67-70}$.
In this scenario, the production of public goods is dependent on a fixed or threshold number of cooperators. In such social dilemmas, each individual in a community would rather avoid the cost of volunteering alone and will seek to exploit public goods produced by others. However, if a threshold of participants is not reached, public goods are not produced, and every individual in the community then pays a cost that is higher than that of volunteering (the so-called 'volunteer's dilemma' (REFS 67,69)). This key feature of the volunteer's dilemma corresponds well with osmotrophic functions because in many cases a threshold level of enzyme secretion (work) is required to sustainably digest a complex food source, implying that a certain level of participation or a certain number of participants is a prerequisite. A good example of this would be the fungal degradation of wood, which requires threshold levels of heterogeneous populations of both lignin-degrading white rot fungi and cellulose-degrading brown rot fungi to facilitate breakdown ${ }^{71,72}$.

The volunteer's dilemma should therefore maintain cheats at a low relative frequency in natural heterogeneous communities ${ }^{66}$, and such a pattern is indeed consistent with observations from one study, in which it was found that $12 \%$ of S. cerevisiae and $10 \%$ of Saccharomyces paradoxus yeast cells sampled from natural and industrial environments do not cooperate by secreting invertase, suggesting that invertase cheats are maintained at a low relative frequency in these heterogeneous populations ${ }^{73}$. The volunteer's dilemma might therefore be significant in explaining how osmotrophs, such as fungi and pseudofungi, engage in public goods production in the heterogeneous microbial communities in which they live.

In reality, however, natural communities are complex and dynamic, so it is unlikely that one factor alone (be it kin selection, community density, repeated interactions, reciprocal cooperation, punishment or the volunteer's dilemma) is the continuous driver of public goods interactions. It is therefore difficult to weigh the relative importance of these factors, and a dynamic mixture of multiple factors should be considered as potential drivers of public goods interactions in osmotrophic communities.

\section{Glossary \\ Appressoria \\ Specialized infection cells that are used by \\ plant-pathogenic fungi to penetrate the host plant surface using mechanical force and/or enzymatic action to breach the cuticle. \\ Cheats \\ Individuals within a community that do not carry out cooperative behaviours (or that minimize their cooperation) but derive benefit from the work of others. \\ Club goods \\ Public goods that are accessible to select individuals only \\ Kin selection \\ Selection that favours traits because of their beneficial effects on the fitness of relatives. \\ Osmotrophic microorganisms \\ Microorganisms that take up digested or dissolved nutrients by osmosis, often facilitated by transporter proteins to allow molecules to cross the cell membrane. \\ Phagotrophy \\ A process governed by the cytoskeleton and involving membrane and cytoplasmic manipulation to engulf large particles or other cells for nutrition.} ('members of the club') in the community.

Cooperators

Individuals that provide benefit to others.

Haustoria

Specialized fungal feeding structures that are commonly produced by biotrophic fungi and occupy living plant cells by invagination of the plant plasma membrane.

\section{Horizontal gene transfer}

The transfer of genetic material between genomes (for example, across species boundaries). Also called lateral gene transfer

\section{Hyphae}

Cells of a filamentous morphotype, sometimes forming branching structures; this morphotype exists for fungi and some other microorganisms. The development of this cellular morphology is governed by the cytoskeleton, with growth and trophic activity directed to the hyphal tip.

Inclusive fitness

The result of individual behaviours on the reproductive output of others, weighted by relatedness.
Private goods

Biological or chemical resources that are produced by an individual and can be used only by that individual.

Public goods

Biological or chemical resources that are produced by an individual in a community and can be used by all other individuals in the community.

\section{Relatedness}

A measure of genetic or genomic similarity.

\section{Rhizoid structures}

'Hair-like' protruberances of eukaryotic cells that maximize the interface between the cell surface and the environment.

Siderophores

Small-molecule iron-chelating compounds that are secreted by microorganisms.

Spiteful behaviour

Behaviour that is costly to both the producer and the recipient. 


\section{HGT can drive public goods interactions}

HGT has been proposed to provide a means by which microorganisms can rapidly gain new biological functions ${ }^{74-77}$. A recent, but steadily growing, body of data suggests that HGT has had a minor role in terms of total gene numbers within microbial eukaryotes, but a much more important role in terms of phenotypic change in the evolution of osmotrophic microorganisms ${ }^{74-76,78}$. An interesting hypothesis has also emerged regarding the role of HGT in determining public goods interactions in populations of pathogenic bacteria $^{5}$. Using a mathematical model, it has been predicted that selection can favour HGT-mediated reintroduction of public goods-encoding genes into cheats ${ }^{5}$.

Further evidence is provided by a study that focused on genes encoding secreted and outer-membrane proteins in 21 Escherichia spp., and looked at the role of HGT in relation to public goods interactions ${ }^{4}$. These proteins might be significant in public goods interactions because they allow different substrates to be utilized, act as effectors to subvert host immune responses and protect the wider microbial community, or contribute to the manufacture of biofilms ${ }^{4}$. Interestingly, genes encoding only $3 \%$ of the secreted proteins and $6 \%$ of the outer-membrane proteins were represented in the core Escherichia spp. genome (defined in REF. 4 as gene families conserved in all 21 bacterial genomes analysed). By contrast, genes encoding inner-membrane, periplasmic space and cytoplasmic proteins are represented within the core genome at much higher proportions $(\sim 24 \%$; $P<0.0001)$. This result is consistent with genes encoding extracellular proteins being frequently lost and acquired by HGT, therefore driving the potential spread of public goods traits within the wider population ${ }^{4,5}$. However, such traits are also likely to be under differing patterns of selection because of their importance for host interactions, resulting in a range of distinct selection pressures that is likely to drive high rates of gene variation, loss, acquisition and replacement.

In this example, additional biological mechanisms are also important for ensuring transfer and maintenance of public goods traits. This study demonstrates that the mobile genetic elements responsible for the transfer of genes associated with public goods traits can enforce cooperative traits by creating 'cellular addiction' processes such as toxin-antitoxin and restriction-modification systems ${ }^{4}$. In this way, cheats within a population can be converted into faithful public goods producers ${ }^{4}$; the newly acquired phenotypes are unlikely to be lost, because maintenance of the selfish genetic element enforces the public goods trait ${ }^{4,79}$. When considered together, these studies show how HGT can lead to the evolution of cooperative phenotypes within microorganisms ${ }^{4}$, and how HGT can 'short-circuit' patterns of kin selection because of the transfer of compatible cooperative traits between different species.

Following on from these studies, we predict that HGT provides one means by which the spread of public goods traits is facilitated in fungal and oomycete populations. However, comparatively little research has looked at HGT of genes that encode transporter proteins from the perspective of public goods interactions, for example. HGT of transporter-encoding genes is likely to be advantageous for microorganisms that reside in environments where osmotrophy is common, because these genes encode the mechanism by which microorganisms can benefit from the availability of previously inaccessible public goods. The acquisition of new transporter functions by HGT would, for instance, be predicted to confer a strong selective advantage and lead to the evolution of new cheats in a population (FIG. 2C). Consistent with this idea, we have identified numerous published examples of transporter-encoding genes that seem to have been transferred between microbial species by $\mathrm{HGT}^{39,40,80-85}$. Key examples include the transfer of genes encoding high-affinity nitrate transporters ${ }^{40,86}$, ammonium transporters $^{87}$ and fructose transporters ${ }^{88}$ into and between fungi, and the transfer of genes encoding sugar and purine or pyrimidine transporters from fungi to oomycetes ${ }^{80,89}$.

HGT has shaped public goods interactions There are two important factors that can influence the frequency of successfully transferring a gene function by HGT. The first factor is whether the gene can be transcribed and translated in a foreign cell, and whether the protein product can fit into the functional network of that cell. The second factor is whether the gene acquisition then provides a selective advantage that leads to maintenance (fixation) of the gene within the recipient species. The first consideration suggests that HGT will occur at a higher frequency for 'operational' genes, which encode housekeeping functions or individual enzymes, than for 'informational' genes, which are involved in transcription, translation and signal transduction. This is because informational genes encode proteins that are typically members of large, complex multiprotein systems. This prediction has therefore been referred to as the complexity hypothesis ${ }^{90}$. According to this hypothesis, it is less likely that HGT of informational genes will introduce novel biological functions ${ }^{90,91}$ which confer a clear selective advantage to a recipient, and it is also less likely that an HGT-acquired informational protein will fit into the functional networks of the recipient cell owing to the requirement for specific interactions with multiple foreign proteins $^{90,91}$. It has recently been shown, however, that the key factor determining the frequency of HGT is not the biological function of a gene family, but rather the connectivity of the encoded proteins, in terms of protein-protein interactions ${ }^{91}$.

We were interested in investigating whether genes involved in the production and acquisition of public goods by osmotrophic microorganisms also have low levels of protein-protein interaction connectivity, suggesting that they therefore have a greater propensity for HGT. We focused on the S. cerevisiae S288c genome, for which a large proportion of gene products have protein interaction data available from twohybrid screens ${ }^{92}$, gene deletion studies ${ }^{93}$ and a collated database of physical and genetic interactions ${ }^{94,95}$, and we investigated those genes $(n=5,084)$ that have been classified as operational or informational, or that are previously identified HGT candidates ${ }^{76}$. We also classified osmotrophy-associated genes encoding transporter proteins and secreted enzymes (FIG. 3). We are aware that analysis of such a data set does not represent a comprehensive analysis of protein interactions, but the yeast protein-protein interaction studies represent the most developed data sets currently available. Interestingly, these data suggest that the connectivity is comparatively low for proteins encoded by the previously identified HGT candidates ${ }^{76}$ and for osmotrophy-associated gene products, consistent with the hypothesis that osmotrophy-associated genes are likely to be successful HGT candidates for trait acquisition.

Next, we collated all published examples of fungal ${ }^{76,87}$ and oomycete ${ }^{89,96}$ genes that were acquired by HGT and encode secreted depolymerizing enzymes (Supplementary information S1 (table)) and transporter proteins (Supplementary information S2 (table)). These lists are derived from a larger collated list of previously published and revalidated HGT studies in fungi (a total of 323 genes $^{76,87}$ ), recent studies focusing on HGT of fructose transporter genes ${ }^{88}$ and ammonium transporter genes ${ }^{87}$ into fungi (ten and two transfers, respectively), and 


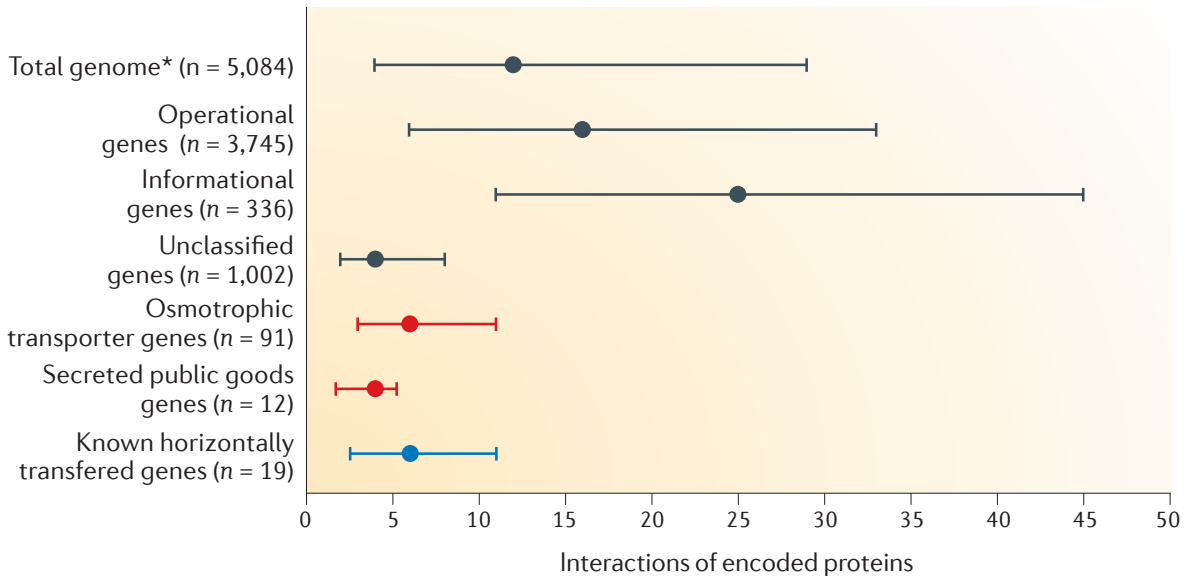

Figure 3 | Comparison of protein-protein interaction conmplexity between horizontally transferred genes and osmotrophic genes in the Saccharomyces cerevisiae S288c genome. Horizontal gene transfer (HGT) events moving genes into the yeast genome have occurred for genes encoding proteins with low levels of connectivity (that is, protein-protein interactions) (blue). Genes classified as functioning in osmotrophic phenotypes, encoding either secreted proteins that function in public goods production or transporter proteins that function in the uptake of public goods (both red), also have a low interaction complexity. This is consistent with such gene classes undergoing HGT. For comparison, we also detail the interaction complexity of the yeast genome as a whole, using the available interaction data. Following the classification used in the original complexity hypothesis ${ }^{90}$, we also show the interaction complexity for proteins encoded by operationa ${ }^{90}$, informational ${ }^{90}$ and unclassified genes. The data are derived from REF. 95. To show the range of complexity, the median and quartile ranges are shown for each category.

individual studies of HGT into oomycetes (a further 35 genes $^{89,96}$ ), giving 370 genes in total. Genes encoding 28 secreted enzymes and 24 metabolite uptake transporters were identified among the HGT candidates, providing evidence that HGT has played a part in the evolution of osmotrophy and of public goods interactions in these groups of microorganisms. It is not possible to state with certainty that HGT has made a significant contribution to the total repertoire of osmotrophy-related gene functions, without functional analyses of the complete proteome and more comprehensive evolutionary analyses. Such analyses would be further complicated because sometimes the relationship between a single gene and a new phenotype is not completely straightforward. For example, acquisition of a metabolite uptake transporter can compensate for previous loss of an entire biosynthetic pathway, as repeatedly seen for folate biosynthesis and salvage (for instance, see REF. 97). However, putative functional annotation of the genes we identified (Supplementary information S1,S2 (tables)) demonstrates that HGT candidates include genes which would significantly expand the nutrient resources available to osmotrophic microorganisms, suggesting that the acquisition of these genes would be selectively advantageous, as predicted by the models illustrated in FIG. 2b,c.
Many of the HGT acquisitions listed in Supplementary information S1,S2 (tables) are therefore likely to be retained by selection primarily because they equip the recipient species with new metabolic capabilities. For example, HGT acquisitions that enable oomycetes to breakdown cutin ${ }^{96}$ or other structural components of plant cell walls ${ }^{89}$ might have provided new mechanisms for these microorganisms to invade their plant hosts. However, these enzymes also release nutrients at the leaf surface as public goods, thereby affecting microbial interactions within the phylloplane community. Therefore, although selection for a horizontally transferred gene might not be driven by public goods interactions initially, these genes nevertheless influence such interactions.

\section{Does HGT shape additional relevant traits?} A key prerequisite for any organism involved in public goods interactions is the capacity to colonize the environment in which particular public goods are located. Hamilton suggested that evolution will act to drive diversification so that strategies evolve to exclude cheats and favour $\mathrm{kin}^{47,48}$. Social theory therefore predicts that when social interactions over public goods are asymmetrical - a situation in which one player produces public goods but has no information to control which organisms benefit from those goods - an interaction can be sustained if there is a cost to a secondary agent entering the environment ${ }^{69}$. If the rewards and the costs to each contributor are optimized appropriately, only preferred agents can enter such interactions, so that potential mutualistic symbionts or kin are favoured, depending on their own capabilities. The principal contributor can therefore control which organisms can engage in an interaction or an environment ${ }^{69}$.

The use of anti-competitor strategies, such as the secretion of toxins, is a policing action that seems to be common among microorganisms $\mathrm{s}^{35,36}$ and is also an example of a public goods interaction because it is a costly behaviour that harms competitors $^{37}$. Such 'spiteful behaviour' results in the exclusion of competitors, but only if the spiteful organisms have the capability to resist their own spiteful behaviour (for example, detoxification or tolerance to toxins $)^{35,98}$. Interestingly, in microorganisms, multiple social traits can be expressed simultaneously $y^{99,100}$ so that public goods production is often combined with spiteful actions such as toxin production ${ }^{35}$. Many fungal and bacterial species, for example, produce a large diversity of toxins and an array of secondary metabolites ${ }^{18,101}$, many of which have no currently known function. Toxins can be used to attack host organisms (by pathogens), to resist predation or to control microorganisms that are competitors in the environment ${ }^{101,102}$. Toxin production can therefore be seen as a key strategy in the protection of public goods by allowing mutualistic symbionts or kin to proliferate and excluding cheats or competitors in an ecosystem where public goods are available ${ }^{101}$.

On the basis of this idea, we investigated whether HGT has played a part in reconfiguring the fungal repertoire of toxins and detoxifying secondary metabolites. There are 27 published examples of phylogenetic data demonstrating HGT events for the transfer of toxin and detoxifying genes into fungal genomes $^{76}$ (Supplementary information S3 (table)) and one additional example identified using genome content comparisons $\mathrm{s}^{103}$ (making 28 in total). Interestingly, of the ten examples of published HGT events between fungi ${ }^{76,103}$, four of the events transferred whole toxin-detoxification gene clusters $^{104,105}$. Furthermore, because many of these 28 HGT events involve gene clusters, these events led to the transfer of a total of 69 individual genes from the above- 
mentioned list of 323 published gene

transfers into fungal genomes ${ }^{76}$.

Arguably, the best example of an HGT of this kind is the transfer of the 23-gene sterigmatocystin cluster (which is important in the manufacture of the toxin sterigmatocystin, related to the carcinogenic aflatoxins) from the Aspergillus clade to Podospora anserina ${ }^{105}$, a fungus that often shares the same environment as Aspergillus spp. This acquisition might have allowed $P$. anserina to gain an advantage in excluding other competitors from the environment and allowed its cohabitation with Aspergillus spp. ${ }^{105}$. HGTmediated acquisitions of genes involved in detoxification metabolic pathways might also aid cheats to subvert kin selection mechanisms and gain entry to an osmotrophic environment where public goods are available. In addition, the requirement for fungi to engage in competition over public goods must therefore have been a factor in the diversification of fungal secondary metabolism ${ }^{18,23,101}$, consistent with the proposal by Hamilton that selection will act to drive diversification of systems that control access to cooperative benefits, such as public goods ${ }^{47,48}$.

\section{Conclusions}

Osmotrophic microorganisms engage in public goods interactions as a product of their mechanism of feeding. Therefore, the acquisition of secreted depolymerizing enzymes, transporter proteins and metabolic pathways associated with toxin biogenesis and detoxification is likely to have been pivotal in the diversification of osmotrophic microorganisms. Consequently, the acquisition of such traits by HGT is likely to have been under strong selective pressure, as it allows recipients to spread to new environments and utilize new food sources. A review of published reports of HGT candidates demonstrates that genes encoding secreted enzymes (28 HGT events or genes), transporters (24 HGT events or genes) and toxin biosynthesis systems (28 HGT events transferring a total of 69 genes) have all been subjected to HGT into osmotrophic eukaryotic microorganisms, such as fungi and oomycetes (see Supplementary information S1-S3 (tables) and REFS 87,88). This group of $121 \mathrm{HGT}$ candidates predicted to function in osmotrophic and public goods phenotypes represents more than $32 \%$ of the 370 collated HGTs into fungi ${ }^{76,87,88}$ and oomycetes $^{89,96}$. We therefore propose that HGT has played a part in the generation, protection and acquisition of public goods by osmotrophic eukaryotes. This hypothesis and the observations reported here fit into a growing body of work ${ }^{1-5}$ which links mechanisms that drive genome variation with the evolution of social traits in microorganisms ${ }^{1}$.

Thomas A. Richards and Nicholas J. Talbot are at the School of Biosciences, University of Exeter, Geoffrey Pope Building, Exeter EX4 4QD, UK.

Thomas A. Richards is also at the Department of Zoology, The Natural History Museum, Cromwell Road, London SW7 5BD, UK.

Correspondence to N.J.T. e-mail: N.J.Talbot@exeter.ac.uk doi: $10.1038 /$ nrmicro3108 Published online 10 September 2013

1. Morris, J. J., Lenski, R. E. \& Zinser, E. R. The Black Queen Hypothesis: evolution of dependencies through adaptive gene loss. mBio 3, e00036-12 (2012).

2. Cordero, O. X., Ventouras, L. A., DeLong, E. F. \& Polz, M. F. Public good dynamics drive evolution of iron acquisition strategies in natural bacterioplankton populations. Proc. Natl Acad. Sci. USA 109, 20059-20064 (2012)

3. Lee, W., van Baalen, M. \& Jansen, V. A. An evolutionary mechanism for diversity in siderophoreproducing bacteria. Ecol. Lett. 15, 119-125 (2012).

4. Nogueira, T. et al. Horizontal gene transfer of the secretome drives the evolution of bacterial cooperation and virulence. Curr. Biol. 19, 1683-1691 (2009).

5. Smith, J. The social evolution of bacterial pathogenesis. Proc. Biol. Sci. 268, 61-69 (2001).

6. Berbee, M. L. \& Taylor, J. W. Dating the molecular clock in fungi - how close are we? Fungal Biol. Rev. 24, 1-16 (2011).

7. Selosse, M. A. \& Le Tacon, F. The land flora: a phototroph-fungus partnership? Trends Ecol. Evol. 13, 15-20 (1998)

8. Taylor, J. W. \& Berbee, M. L. Dating divergences in the fungal tree of life: review and new analyses. Mycologia 98, 838-849 (2006).

9. Pirozynski, K. A. \& Malloch, D. W. The origin of land plants: a matter of mycotrophism. Biosystems 5 , 153-164 (1975)

10. Fisher, M. C. et al. Emerging fungal threats to animal, plant and ecosystem health. Nature 484, 186-194 (2012).

11. Hawksworth, D. L. The fungal dimension of biodiversity: magnitude, significance and conservation. Mycol. Res. 95, 641-655 (1991).

12. Hibbett, D. S. et al. A higher-level phylogenetic classification of the Fungi. Mycol. Res. 111, 509-547 (2007).

13. James, T. Y. et al. Reconstructing the early evolution of Fungi using a six-gene phylogeny. Nature 443 , 818-822 (2006).

14. Richards, T. A., Jones, M. D. M., Leonard, G. \& Bass, D. Marine fungi: their ecology and molecular diversity. Annu. Rev. Mar. Sci. 4, 495-522 (2012).

15. Buée, M. et al. 454 Pyrosequencing analyses of forest soils reveal an unexpectedly high fungal diversity. New Phytol. 184, 449-456 (2009).

16. Jumpponen, A. \& Jones, K. L. Massively parallel 454 sequencing indicates hyperdiverse fungal communities in temperate Quercus macrocarpa phyllosphere. New Phytol. 184, 438-448 (2009).

17. O'brien, H. E., Parrent, J. L., Jackson, J. A., Moncalvo, J.-M. \& Vilgalys, R. Fungal community analysis by large-scale sequencing of environmental samples. Appl. Environ. Microbiol. 71, 5544-5550 (2005).

18. Keller, N. P., Turner, G. \& Bennett, J. W. Fungal secondary metabolism - from biochemistry to genomics. Nature Rev. Microbiol. 3, 937-947 (2005)

19. Stajich, J. E. et al. The fungi. Curr. Biol. 19, R840-R845 (2009).

20. Soanes, D. M. et al. Comparative genome analysis of filamentous fungi reveals gene family expansions associated with fungal pathogenesis. PLOS ONE 3 , e2300 (2008).

21. Cornell, M. J. et al. Comparative genome analysis across a kingdom of eukaryotic organisms: specialization and diversification in the fungi. Genome Res. 17, 1809-1822 (2007).

22. Chiang, Y. M., Lee, K. H., Sanchez, J. F., Keller, N. P. \& Wang, C. C. Unlocking fungal cryptic natural products. Nat. Prod. Commun. 4, 1505-1510 (2009).
23. Bushley, K. E. \& Turgeon, B. G. Phylogenomics reveals subfamilies of fungal nonribosomal peptide synthetases and their evolutionary relationships. BMC Evol. Biol. 10, 26 (2010).

24. Amselem, J. et al. Genomic analysis of the necrotrophic fungal pathogens Sclerotinia sclerotiorum and Botrytis cinerea. PLoS Genet. 7, e1002230 (2011)

25. de Boer, W., Folman, L. B., Summerbell, R. C. \& Boddy, L. Living in a fungal world: impact of fungi on soil bacterial niche development. FEMS Microbiol. Rev. 29, 795-811 (2005).

26. Cavalier-Smith, T. in Evolutionary Biology of the Fungi (British Mycological Society Symposia) 339-353 (eds Rayer, A. D. M., Brasier, C. M. \& Moore, D., 1987).

27. Cavalier-Smith, T. \& Chao, E. E. Phylogeny and megasystematics of phagotrophic heterokonts (kingdom Chromista). J. Mol. Evol. 62, 388-420 (2006).

28. Bermudez Moretti, M., Perullini, A. M., Batlle, A. \& Correa Garcia, S. Expression of the UGA4 gene encoding the $\delta$-aminolevulinic and $\gamma$-aminobutyric acids permease in Saccharomyces cerevisiae is controlled by amino acid-sensing systems. Arch. Microbiol. 184, 137-140 (2005).

29. Boles, E. \& Hollenberg, C. P. The molecular genetics of hexose transport in yeasts. FEMS Microbiol. Rev. 21, 85-111 (1997)

30. Heymann, P., Ernst, J. F. \& Winkelmann, G Identification of a fungal triacetylfusarinine $C$ siderophore transport gene (TAF1) in Saccharomyces cerevisiae as a member of the major facilitator superfamily. Biometals 12, 301-306 (1999).

31. Gore, J., Youk, H. \& van Oudenaarden, A. Snowdrift game dynamics and facultative cheating in yeast. Nature 459, 253-256 (2009).

32. Greig, D. \& Travisano, M. The Prisoner's Dilemma and polymorphism in yeast SUC genes. Proc. Biol. Sci. 27 (Suppl. 3), S25-S26 (2004).

33. Crespi, B. J. The evolution of social behavior in microorganisms. Trends. Ecol. Evol. 16, 178-183 (2001).

34. West, S. A., Diggle, S. P., Buckling, A., Gardner, A. \& Griffin, A. S. The social lives of microbes. Annu. Rev. Ecol. Evol. Syst. 38, 53-77 (2007).

35. Inglis, R. F., Brown, S. P. \& Buckling, A. Spite versus cheats: competition among social strategies shapes virulence in Pseudomonas aeruginosa. Evolution 66, 3472-3484 (2012).

36. Riley, M. A. $\&$ Wertz, J. E. Bacteriocins: evolution, ecology, and application. Annu. Rev. Microbiol. 56, 117-137 (2002)

37. West, S. A., Griffin, A. S. \& Gardner, A. Social semantics: altruism, cooperation, mutualism, strong reciprocity and group selection. J. Evol. Biol. 20 415-432 (2007)

38. MaClean, R. C., Fuentes-Hernandez, A., Greig, D. Hurst, L. D. \& Gudelj, I. A mixture of "cheats" and "co-operators" can enable maximal group benefit. PLoS Biol. 8, e1000486 (2010).

39. Galeote, V. et al. FSY1, a horizontally transferred gene in the Saccharomyces cerevisiae EC1118 wine yeast strain, encodes a high-affinity fructose $/ \mathrm{H}^{+}$symporter. Microbiology 156, 3754-3761 (2011).

40. Slot, J. C. \& Hibbett, D. S. Horizontal transfer of a nitrate assimilation gene cluster and ecological transitions in fungi: a phylogenetic study. PLOS ONE 2 e1097 (2007).

41. Brown, C. J., Todd, K. M. \& Rosenzweig, R. F. Multiple duplications of yeast hexose transport genes in response to selection in a glucose-limited environment. Mol. Biol. Evol. 15, 931-942 (1998).

42. Wei, H. et al. A putative high affinity hexose transporter, $h x t A$, of Aspergillus nidulans is induced in vegetative hyphae upon starvation and in ascogenous hyphae during cleistothecium formation. Fungal Genet. Biol. 41, 148-156 (2004).

43. Javelle, A., Andre, B., Marini, A.-M. \& Chalot, M. Highaffinity ammonium transporters and nitrogen sensing in mycorrhizas. Trends Microbiol. 11, 53-55 (2003).

44. Smith, E. E., Sims, E. H., Spencer, D. H., Kaul, R. \& Olson, M. V. Evidence for diversifying selection at the pyoverdine locus of Pseudomonas aeruginosa. J. Bacteriol. 187, 2138-2147 (2005).

45. Kruckeberg, A. L. The hexose transporter family of Saccharomyces cerevisiae. Arch. Microbiol. 166, 283-292 (1996)

46. Koschwanez, J. H., Foster, K. R. \& Murray, A. W Sucrose utilization in budding yeast as a model for the origin of undifferentiated multicellularity. PLOS Biol. 9 e1001122 (2011) 
47. Hamilton, W. D. The genetical evolution of social behaviour, I. J. Theor. Biol. 7, 1-16 (1964).

48. Hamilton, W. D. The genetical evolution of social behaviour, II. J. Theor. Biol. 7, 17-52 (1964).

49. Griffin, A. S., West, S. A. \& Buckling, A. Cooperation and competition in pathogenic bacteria. Nature 430 1024-1027 (2004).

50. Nadell, C. D., Foster, K. R. \& Xavier, J. B. Emergence of spatial structure in cell groups and the evolution of cooperation. PLoS Comput. Biol. 6, e 1000716 (2010).

51. Nowak, M. A. \& May, R. M. Evolutionary games and spatial chaos. Nature 359, 826-829 (1992).

52. Hauert, C. \& Doebeli, M. Spatial structure often inhibits the evolution of cooperation in the snowdrift game. Nature 428, 643-646 (2004)

53. Kummerli, R., Griffin, A. S., West, S. A., Buckling, A. \& Harrison, F. Viscous medium promotes cooperation in the pathogenic bacterium Pseudomonas aeruginosa. Proc. Biol. Sci. 276, 3531-3538 (2009).

54. Kummerli, R., Gardner, A., West, S. A. \& Griffin, A. S. Limited dispersal, budding dispersal, and cooperation: an experimental study. Evolution 63, 939-949 (2009).

55. Brockhurst, M. A Buckling A \& Gardner A Cooperation peaks at intermediate disturbance. Curr. Biol. 17, 761-765 (2007)

56. Verbruggen, E. et al. Spatial structure and interspecific cooperation: theory and an empirical test using the mycorrhizal mutualism. Am. Nat. 179, E133-E146 (2012).

57. Doolittle, W. F. Microbial evolution: stalking the wild bacterial species. Curr. Biol. 18, R565-567 (2008).

58. Boenigk, J., Ereshefsky, M., Hoef-Emden, K., Mallet, J. $\&$ Bass, D. Concepts in protistology: species definition and boundaries. Eur. J. Protistol. 48, 96-102 (2012).

59. Taylor, J. W. et al. Phylogenetic species recognition and species concepts in fungi. Fungal Genet. Biol. 31 , 21-32 (2000)

60. Trivers, R. L. The evolution of reciprocal altruism Q. Rev. Biol. 46, 37-57 (1971).

61. Axelrod, R. \& Hamilton, W. D. The evolution of cooperation. Science 211, 1390-1396 (1981)

62. Clutton-Brock, T. H. \& Parker, G. A. Punishment in animal species. Nature 373, 209-216 (1995)

63. Fehr, E. \& Gächter, S. Altruistic punishment in humans. Nature 415, 137-140 (2002).

64. Kiers, E. T. et al. Reciprocal rewards stabilize cooperation in the mycorrhizal symbiosis. Science 333, 880-882 (2011).

65. Kiers, E. T., Rousseau, R. A., West, S. A. \& Denison, R. F. Host sanctions and the legumerhizobium mutualism. Nature 425, 78-81 (2003).

66. Hauert, C., Holmes, M. \& Doebeli, M. Evolutionary games and population dynamics: maintenance of cooperation in public goods games. Proc. Biol. Sci. 273, 2565-2570 (2006)

67. Archetti, M. \& Scheuring, I. Coexistence of cooperation and defection in public goods games. Evolution 65, 1140-1148 (2011).

68. Raihani, N. J. \& Bshary, R. The evolution of punishment in $n$-player public goods games: a volunteer's dilemma. Evolution 65, 2725-2728 (2011).

69. Archetti, M. et al. Economic game theory for mutualism and cooperation. Ecol. Lett. 14, 1300-1312 (2011).

70. Hauert, C., De Monte, S., Hofbauer, J. \& Sigmund, K. Volunteering as Red Queen mechanism for cooperation in public goods games. Science $\mathbf{2 9 6}$ 1129-1132 (2002).

71. Floudas, D. et al. The Paleozoic origin of enzymatic lignin decomposition reconstructed from 31 fungal genomes. Science 336, 1715-1719 (2012).
72. Hastrup, A. C. S. et al. Differences in crystalline cellulose modification due to degradation by brown and white rot fungi. Fungal Biol. 116, 1052-1063 (2012).

73. Maclean, C. R. \& Brandon, C. Stable public goods cooperation and dynamic social interactions in yeast. J. Evol. Biol. 21, 1836-1843 (2008).

74. Garcia-Vallve, S., Romeu, A. \& Palau, J. Horizontal gene transfer of glycosyl hydrolases of the rumen fungi. Mol. Biol. Evol. 17, 352-361 (2000).

75. Gojkovic, Z. et al. Horizontal gene transfer promoted evolution of the ability to propagate under anaerobic conditions in yeasts. Mol. Genet. Genom. 271, 387-393 (2004).

76. Richards, T. A., Leonard, G., Soanes, D. M. \& Talbot, N. J. Gene transfer into the fungi. Fungal Biol. Rev. 25, 98-110 (2011).

77. Jain, R., Rivera, M. C., Moore, J. E. \& Lake, J. A. Horizontal gene transfer accelerates genome innovation and evolution. Mol. Biol. Evol. 20 , 1598-1602 (2003).

78. Gabaldon, T. \& Huynen, M. A. Reconstruction of the proto-mitochondrial metabolism. Science 301, 609 (2003).

79. Lawrence, J. G. Microbial evolution: enforcing cooperation by partial kin selection. Curr. Biol. 19 , R943-R945 (2009).

80. Richards, T. A., Dacks, J. B., Jenkinson, J. M., Thornton, C. R. \& Talbot, N. J. Evolution of filamentous plant pathogens: gene exchange across eukaryotic kingdoms. Curr. Biol. 16, 1857-1864 (2006).

81. Richards, T. A. et al. Phylogenomic analysis demonstrates a pattern of rare and ancient horizontal gene transfer between plants and fungi. Plant Cell 21, 1897-1911 (2009)

82. Noll, K. M \& Thirangoon, K. Interdomain transfers of sugar transporters overcome barriers to gene expression. Methods Mol. Biol. 532, 309-322 (2009).

83. Hellborg, L., Woolfit, M., Arthursson-Hellborg, M. \& Piskur, J. Complex evolution of the DAL5 transporter family. BMC Genomics 9, 164 (2008).

84. Gomolplitinant, K. M. \& Saier, M. H. Jr. Evolution of the oligopeptide transporter family. J. Membr. Biol. 240, 89-110 (2011)

85. Nesbo, C. L., Nelson, K. E. \& Doolittle, W. F. Suppressive subtractive hybridization detects extensive genomic diversity in Thermotoga maritima J. Bacteriol. 184, 4475-4488 (2002).

86. Slot, J. C., Hallstrom, K. N., Matheny, P. B. \& Hibbett, D. S. Diversification of NRT2 and the origin of its fungal homolog. Mol. Biol. Evol. 24, 1731-1743 (2007).

87. McDonald, T. R., Dietrich, F. S. \& Lutzoni, F. Multiple horizontal gene transfers of ammonium transporters ammonia permeases from prokaryotes to eukaryotes: towards a new functional and evolutionary classification. Mol. Biol. Evol. 29, 51-60 (2012)

88. Coelho, M. A. Gonçalves, C., Sampaio, J. P. \& Gonçalves, P. Extensive intra-kingdom horizontal gene transfer converging on a fungal fructose transporter gene. PLoS Genet. 9, e 1003587 (2013).

89. Richards, T. A. et al. Horizontal gene transfer facilitated the evolution of plant parasitic mechanisms in the oomycetes. Proc. Natl Acad. Sci. USA 108 15258-15263 (2011)

90. Jain, R., Rivera, M. C. \& Lake, J. A. Horizontal gene transfer among genomes: the complexity hypothesis. Proc. Natl Acad. Sci. USA 96, 3801-3806 (1999).

91. Cohen, O., Gophna, U. \& Pupko, T. The complexity hypothesis revisited: connectivity rather than function constitutes a barrier to horizontal gene transfer. $\mathrm{Mol}$. Biol. Evol. 28, 1481-1489 (2011).

92. Uetz, P. et al. A comprehensive analysis of proteinprotein interactions in Saccharomyces cerevisiae. Nature 403, 623-627 (2000).
93. Jeong, H., Mason, S. P., Barabasi, A. L. \& Oltvai, Z. N Lethality and centrality in protein networks. Nature 411, 41-42 (2001)

94. Stark, C. et al. BioGRID: a general repository for interaction datasets. Nucleic Acids Res. 34 D535-539 (2006).

95. Cotton, J. A. \& Mclnerney, J. O. Eukaryotic genes of archaebacterial origin are more important than the more numerous eubacterial genes, irrespective of function. Proc. Natl Acad. Sci. USA 107. 17252-17255 (2010)

96. Belbahri, L., Calmin, G., Mauch, F. \& Andersson, J. O Evolution of the cutinase gene family: evidence for lateral gene transfer of a candidate Phytophthora virulence factor. Gene 408, 1-8 (2008).

97. de Crecy-Lagard, V., El Yacoubi, B., de la Garza, R. D. Noiriel, A. \& Hanson, A. D. Comparative genomics of bacterial and plant folate synthesis and salvage: predictions and validations. BMC Genomics 8, 245 (2007).

98. Lehmann, L., Bargum, K. \& Reuter, M. An evolutionary analysis of the relationship between spite and altruism. J. Evol. Biol. 19, 1507-1516 (2006).

99. Harrison, F. \& Buckling, A. Siderophore production and biofilm formation as linked social traits. ISME J. 3 , 632-634 (2009).

100. Williams, P., Winzer, K., Chan, W. C. \& Camara, M Look who's talking: communication and quorum sensing in the bacterial world. Phil. Trans. R. Soc. B 362, 1119-1134 (2007).

101. Fox, E. M. \& Howlett, B. J. Secondary metabolism: regulation and role in fungal biology. Curr. Opin. Microbiol. 11, 481-487 (2008).

102. Trejo-Estrada, S. R., Paszczynski, A. \& Crawford, D. L. Antibiotics and enzymes produced by the biocontrol agent Streptomyces violaceusniger YCED-9. J. Ind. Microbiol. Biotechnol. 21, 81-90 (1998).

103. Friesen, T. L. et al. Emergence of a new disease as a result of interspecific virulence gene transfer. Nature Genet. 38, 953-956 (2006).

104. Patron, N. J. et al. Origin and distribution of epipolythiodioxopiperazine (ETP) gene clusters in filamentous ascomycetes. BMC Evol. Biol. 7, 174 (2007).

105. Slot, J. C. \& Rokas, A. Horizontal transfer of a large and highly toxic secondary metabolic gene cluster between fungi. Curr. Biol. 21, 134-139 (2011)

106. Powell, M. J., Letcher, P. M. \& Longcore, J. E. Pseudorhizidium is a new genus with distinct zoospore ultrastructure in the order Chytridiales. Mycologia 105, 496-507 (2013).

\section{Acknowledgements}

T.A.R. is a European Molecular Biology Organization Young Investigator and a Leverhulme Early Career Fellow, and his research group is supported by grants from the Moore Foundation, the UK Natural Environment Research Council and the Biotechnology and Biological Sciences Research Council (BBSRC). N.J.T. is a European Research Council Advanced Investigator and receives funding from the BBSRC, the Bill and Melinda Gates Foundation and the Halpin Trust. The authors thank A. Buckling and the anonymous reviewers for comments on this manuscript.

Competing interests statement

The authors declare no competing financial interests.

SUPPLEMENTARY INFORMATION

See online article: $\mathbf{S 1}$ (table) $\mid \mathrm{S} 2$ (table) $\mid \mathrm{S} 3$ (table) ALL LINKS ARE ACTIVE IN THE ONLINE PDF 